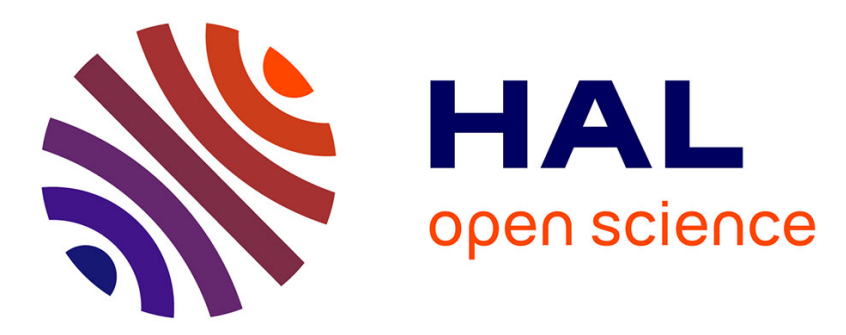

\title{
Observation d'un phénomène de relaxation structurale dans le sélénium vitreux par mesure des propriétés mécaniques
}

\author{
S. Etienne, J. Pérez
}

\section{- To cite this version:}

S. Etienne, J. Pérez. Observation d'un phénomène de relaxation structurale dans le sélénium vitreux par mesure des propriétés mécaniques. Revue de Physique Appliquée, 1979, 14 (5), pp.607-611. 10.1051/rphysap:01979001405060700 . jpa-00244636

\section{HAL Id: jpa-00244636 https://hal.science/jpa-00244636}

Submitted on 1 Jan 1979

HAL is a multi-disciplinary open access archive for the deposit and dissemination of scientific research documents, whether they are published or not. The documents may come from teaching and research institutions in France or abroad, or from public or private research centers.
L'archive ouverte pluridisciplinaire HAL, est destinée au dépôt et à la diffusion de documents scientifiques de niveau recherche, publiés ou non, émanant des établissements d'enseignement et de recherche français ou étrangers, des laboratoires publics ou privés. 


\title{
Observation d'un phénomène de relaxation structurale dans le sélénium vitreux par mesure des propriétés mécaniques
}

\author{
S. Etienne et J. Pérez \\ Groupe d'Etudes de Métallurgie Physique et de Physique des Matériaux, E.R.A. 463, I.N.S.A. de Lyon, \\ 20, avenue Albert Einstein, 69621 Villeurbanne Cedex, France
}

(Reçu le 13 août 1978, révisé le 22 janvier 1979, accepté le 29 janvier 1979)

\begin{abstract}
Résumé. - Un effet de relaxation structurale dans le sélénium vitreux massif a déjà été mis en évidence par des techniques calorimétriques. Nous avons observé un effet de même type en étudiant les propriétés micromécaniques du matériau (fluage et relaxation de contrainte sous faible charge, frottement intérieur) en cours de vieillissement isotherme à une température inférieure ou proche du domaine de transition vitreuse $T_{\mathrm{g}}$. Nous montrons que la théorie du volume libre peut rendre compte qualitativement des résultats expérimentaux sans qu'une modification de la nature ou de la forme des unités structurales soit à priori nécessaire.
\end{abstract}

\begin{abstract}
A structure relaxation effect has been previously observed in the case of bulk amorphous selenium mainly through calorimetric measurements. A similar effect is observed while studying micromechanical properties (creep and stress relaxation under small load, internal friction) during isothermal ageing at a temperature lower or near the glass transition range. It is shown that the free volume theory describes qualitatively the experimental results, any assumption about a modification of the kind or the number of structural units being not necessary.
\end{abstract}

1. Introduction. - Le passage d'un solide vitreux dans la zone de température $T_{\mathrm{g}}$ correspondant à la transition vitreuse s'accompagne d'une modification importante de certaines propriétés du matériau. Dans le cas du sélénium, la résistivité électrique, par exemple, présente une anomalie et un effet d'hystérésis importants à la traversée de ce domaine de température [1]. D'autre part, pour une température donnée, les propriétés observées dépendent fortement de l'histoire thermique [2]. Ainsi dans le cas du sélénium vitreux un effet de relaxation structurale a été observé par calorimétrie $[3,4,5]$. Cornet et Rossier [6] constatent en outre que cet effet de relaxation apparaît à la suite du chauffage du matériau au-delà d'une température (entre 50 et $60^{\circ} \mathrm{C}$ ) où un pic endothermique est observé sur la courbe ATD; ils en déduisent que le sélénium vitreux se polymérise après un tel chauffage : le phénomène de relaxation structurale peut alors être interprété comme une réaction de dépolymérisation.

D'une manière générale les expériences évoquées ci-dessus se rapportent à l'état des liaisons entre les entités structurales constituant le sélénium vitreux il est alors nécessaire de compléter ces études par des mesures de propriétés caractérisant la mobilité de ces entités structurales. Nous nous sommes donc proposés d'effectuer des mesures de propriétés micromécaniques : frottement intérieur à basse fréquence, fluage et relaxation de contrainte. Ces mesures font également apparaître un effet de relaxation structurale ; nous proposons alors une description des résultats obtenus en invoquant simplement la notion de volume libre.

2. Conditions expérimentales. - 2.1 ApPAREILLAGE. - L'échantillon qui se présente sous la forme d'une plaquette de $60 \times 5 \times 1 \mathrm{~mm}^{3}$ constitue l'élément de rappel d'un pendule de torsion. Pour augmenter la quantité d'information présentée par un spectre de frottement intérieur en fonction de la température, on cherche généralement à décaler l'ensemble du spectre vers les basses températures en abaissant la fréquence : nous effectuons donc des mesures avec un pendule pouvant fonctionner aux très basses fréquences $\left(10^{-5}\right.$ à $\left.1 \mathrm{~Hz}\right)$ en régime forcé, son moment d'inertie étant très faible [7].

A l'aide de ce dispositif, les propriétés mécaniques de l'échantillon sont étudiées sous faibles charges; en effet, la déformation relative maximale imposée élastiquement à l'échantillon est de l'ordre de $10^{-5}$. La contrainte appliquée à l'échantillon est soit sinusoïdale (étude du module de cisaillement complexe), soit en échelon (essais de fluage-relaxation, mesure de la viscosité, mise en évidence de l'élasticité retardée).

2.2 Etat du matériau. - Le sélénium, de pureté 5-9 a été obtenu à l'état vitreux par trempe à l'air 

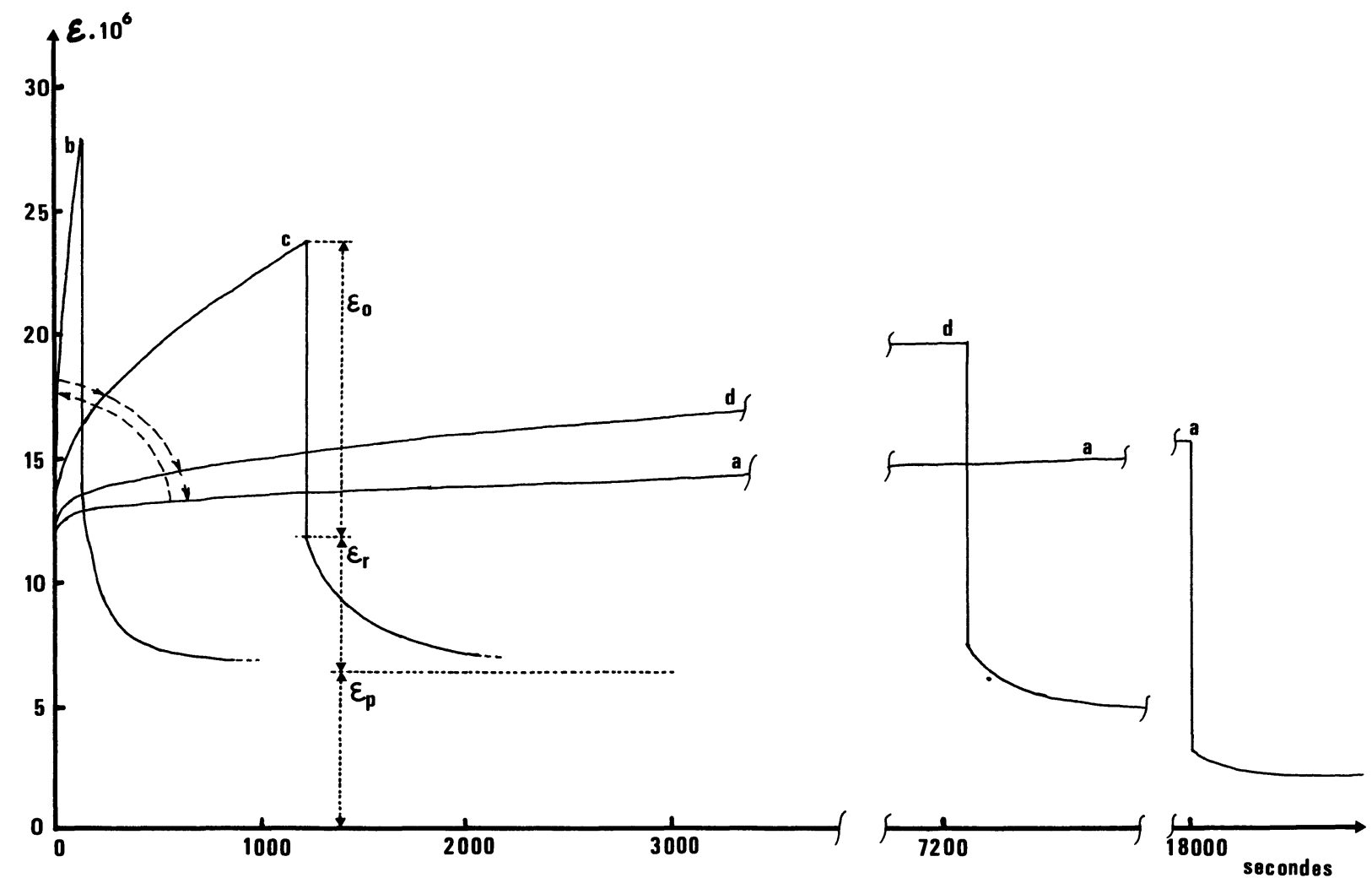

Fig. 1. - Essai de fluage effectué en torsion sur le même échantillon à $17^{\circ} \mathrm{C}$ par différents temps de vieillissement après trempe : $600 \mathrm{~s}(\mathrm{~b})$, $7200 \mathrm{~s}$ (c), $1,3 \times 10^{4} \mathrm{~s}$ (d) et $10^{7} \mathrm{~s}(\mathrm{a})$. La contrainte appliquée est à chaque fois égale à $10^{-5} \mathrm{G}:$ pour cette valeur faible le comportement du matériau est sensiblement newtonien. Les déformations élastiques, recouvrable et permanente sont notées $\varepsilon_{0}, \varepsilon_{\mathrm{r}}$ et $\varepsilon_{\mathrm{p}}$ respectivement.

[Torsional creep test obtained at $17{ }^{\circ} \mathrm{C}$ for various times of ageing after quenching and for the same specimen (the applied stress is about $10^{-5} \mathrm{G}$ : for this law value, the behaviour of the specimen is expected to be newtonian) :600 s (b), $7200 \mathrm{~s}(\mathrm{c}), 1.3 \times 10^{4} \mathrm{~s}(\mathrm{~d}), 10^{7} \mathrm{~s}(\mathrm{a})$. The elastic, recovered and permanent deformation are denoted $\varepsilon_{0}, \varepsilon_{\mathrm{r}}$ and $\varepsilon_{\mathrm{p}}$ respectively.]

à partir de $400^{\circ} \mathrm{C}$ environ. L'examen métallographique permet de vérifier que le nombre de bulles observables (diamètre au moins égal à quelque $10^{-6} \mathrm{~m}$ ) est négligeable. Après avoir été étudié dans l'état vieilli (plusieurs semaines à la température ordinaire), le matériau est porté pendant $300 \mathrm{~s}$ à $70^{\circ} \mathrm{C}$ puis refroidi par trempe à l'eau. Dans la suite de ce travail, nous qualifierons l'état de l'échantillon ainsi traité d'état de base.

3. Résultats expérimentaux. - A l'état vieilli, l'essai de fluage et de relaxation conduit à la courbe a de la figure 1. Il apparaît clairement que la déformation par fluage est faible (environ $2 \times 10^{-6}$ au bout de $2 \times 10^{4} \mathrm{~s}$ ) et, en outre, la partie recouvrable de la déformation reste inférieure à $10^{-6}$. Nous avons ensuite étudié le vieillissement à température ordinaire d'un échantillon à l'état de base.

3.1 Essais De fluage ET DE RelaXation. - Les courbes $b, c, d$ correspondent à trois exemples de réponse de l'échantillon à un créneau de contrainte pour différentes durées de vieillissement. Une analyse quantitative fine nécessiterait l'introduction de distributions dans les temps et les intensités de relaxation ; néanmoins, en première approximation on remarque que dans le cas de l'échantillon dans l'état de base, la viscosité apparente est bien plus faible qu'à l'état vieilli; d'autre part, la composante viscoélastique représentée par la partie recouvrable de la déformation est plus importante.

De plus l'évolution de ces deux grandeurs semblent corrélées au cours du vieillissement. La figure 2

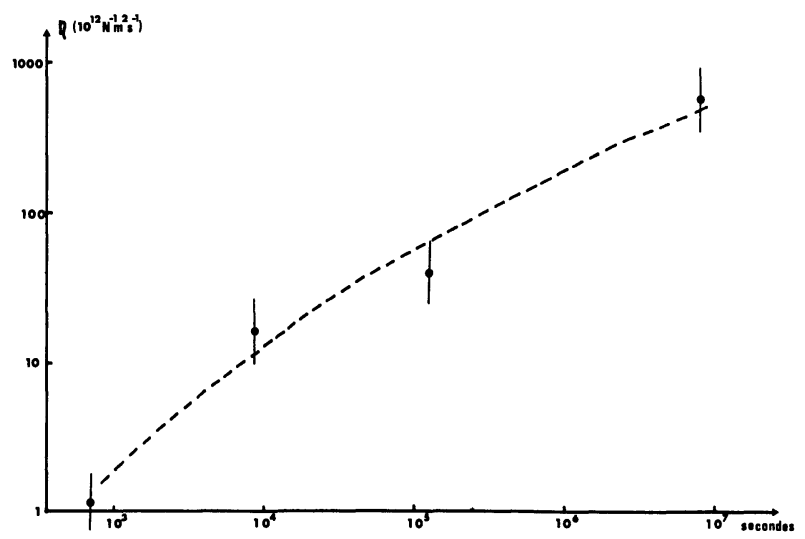

Fig. 2. - Evolution de la viscosité apparente à $17^{\circ} \mathrm{C}$ avec le temps de vieillissement.

[Viscosity behaviour during ageing at $17^{\circ} \mathrm{C}$.] 
représente plus globalement l'évolution de la viscosité apparente (en cisaillement, lors du vieillissement).

3.2 ETUDE DU MODULE DYNAMIQUE COMPLEXE. Afin de préciser les résultats obtenus en fluage et relaxation de contrainte nous avons soumis le matériau à une contrainte sinusoïdale très basse fréquence $(0,008 \mathrm{~Hz})$ et observé l'évolution du module de cisaillement complexe $G^{*}=G \mathrm{e}^{j \varphi}$ que nous caractériserons par son module $G$ et le coefficient de frottement intérieur $Q^{-1}=\operatorname{tg} \varphi$. $G$ et $Q^{-1}$ sont obtenus après traitement numérique des données caractérisant les cycles contrainte-déformation [8]. La période de travail a été choisie de façon à être du même ordre de grandeur que les temps de relaxation observés lors des mesures précédentes, c'est-à-dire en 1 re approximation, le temps pendant lequel s'effectue la recouvrance de la déformation.

Nous avons effectué deux types d'essais :

a) A température constante (Fig. 3) : mesure du module et du coefficient de frottement intérieur en fonction du temps après trempe, mettant en évidence l'évolution des propriétés mécaniques.

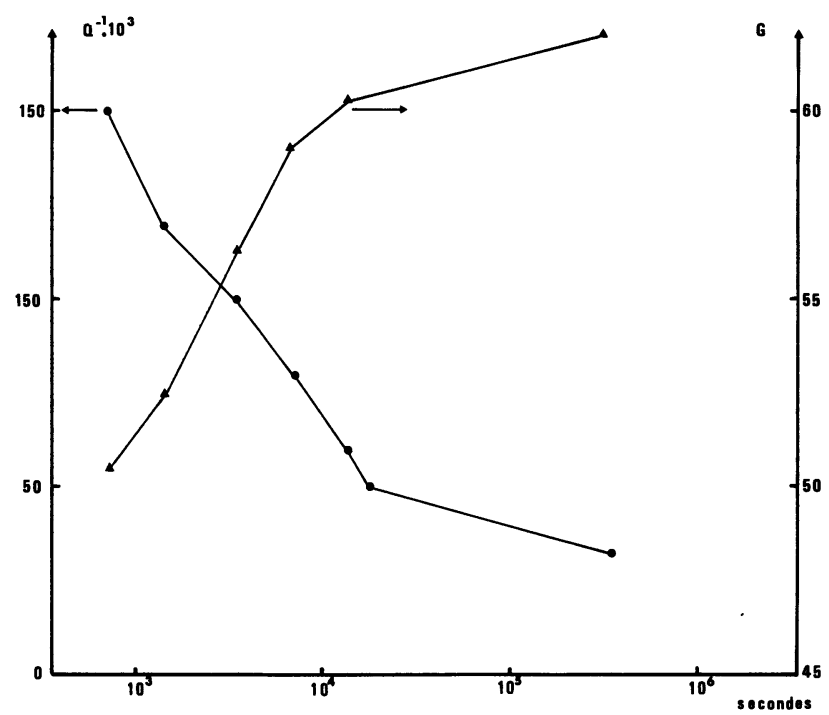

Fig. 3. - Evolution du module $G$ (unité arbitraire) et du coefficient de frottement intérieur $Q^{-1}$ avec le temps de vieillissement à $17^{\circ} \mathrm{C}$. [Evolution of the shear modulus $G$ (arbitrary unit) and of internal friction $Q^{-1}$ during ageing at $17^{\circ} \mathrm{C}$.]

b) A température variable (Fig. 4) : après trempe à $12^{\circ} \mathrm{C}$, les spectres de module et de frottement intérieur ont été tracés tout d'abord au cours du premier refroidissement jusqu'à température de l'azote liquide, pendant lequel la relaxation structurale est figée. Le maintien de la structure au refroidissement est bien confirmé par les spectres $G(T)$ et $Q^{-1}(T)$ obtenus au cours de montées en température successives : il faut en effet atteindre des températures voisines de la température de trempe pour une évolution notable des spectres apparaissant pendant la mesure (valeur plus faible de $Q^{-1}$ et plus élevée pour $G$ à une température donnée).

4. Discussion. - L'évolution des propriétés mécaniques que nous venons de présenter traduisent un effet de relaxation structurale après trempe. Cet effet, peut être décrit en considérant la dépendance très classique des paramètres thermodynamiques d'un matériau vitreux en fonction de l'histoire thermique. Considérons par exemple la variation du volume spécifique $V$ avec la température représentée schématiquement sur la figure 5 [9].

Cette figure nous permet de rappeler les définitions de la zone de transition $T_{\mathrm{g}}$ (domaine séparant la droite caractéristique du liquide surfondu de celle du verre), la température fictive $T_{\mathrm{gf}}$ (intersection des deux droites précédentes extrapolées) et la température $T_{0}$ pour laquelle l'entropie configurationnelle s'annule). A l'aide de cette figure, l'histoire thermique de nos essais se résume ainsi :

a) Chauffage à $70^{\circ} \mathrm{C}$ pendant $300 \mathrm{~s}$ (point A) : une telle durée est supérieure au temps de relaxation structurale [4], mais insuffisante pour entraîner une dévitrification notable comme le montre d'une part



Fig. 4. - Spectre de frottement intérieur et de module présentés sur un même échantillon au cours de montées successives en température jusqu'à : $-5^{\circ} \mathrm{C}(0),+12^{\circ} \mathrm{C}(\bullet),+20^{\circ} \mathrm{C}(\Delta)$, et $+20^{\circ} \mathrm{C}$ (A).

[Internal friction and modulus spectra during successive heating (for the same specimen) up to : $-5^{\circ} \mathrm{C}(0),+12^{\circ} \mathrm{C}(\bullet),+20^{\circ} \mathrm{C}$ $(\Delta)$ and $+20^{\circ} \mathrm{C}(\Delta)$. ] 
les contrôles aux rayons $\mathrm{X}$ et surtout, d'autre part la reproductibilité des courbes $Q^{-1}=f(T)$ après plusieurs trempes à partir de $70^{\circ} \mathrm{C}$ (notons à ce sujet que, si la sensibilité du premier type de contrôle est médiocre, le frottement intérieur varie beaucoup lorsque l'on passe de l'état vitreux à l'état cristallin [7]). Les températures réelles et fictives sont alors confondues.

b) Refroidissement rapide par trempe jusqu'à la température ambiante (point B). La température fictive correspondante et caractérisant l'état de base est ainsi $T_{\mathrm{gf}}(\mathrm{B})$.

c) Vieillissement à température ambiante (point $\mathrm{C}$ ). La température fictive diminue et passe de $T_{\mathrm{gf}}(\mathrm{B})$ à $T_{\mathrm{gf}}(\mathrm{C})$.

Les résultats précédents permettent de comparer, du moins qualitativement, d'une part l'influence de la température fictive $T_{\mathrm{gf}}$ à température réelle $T$ constante (Fig. 3) et d'autre part celle de la température réelle à température fictive constante (Fig. 4). Nous verrons ainsi dans ce paragraphe, qu'une augmentation de $T$ ou de $T_{\mathrm{gf}}$ produisent qualitativement les mêmes effets sur les propriétés; le cas de la viscosité sera plus particulièrement décrit.

En utilisant les notions classiques concernant les solides vitreux on peut considérer que le vieillissement a pour effet de diminuer le volume libre tout comme l'entropie configurationnelle; nous allons donc utiliser le modèle entropique de fluage proposé par Chen [10] et applicable en dessous de la zone de transition vitreuse ; dans le cadre de ce modèle la viscosité est donnée par l'expression :

avec

$$
\eta=\eta_{0} \exp \frac{\Delta H}{R T}
$$

$$
\Delta H=\frac{C R T_{\mathrm{gf}}}{T_{\mathrm{gf}}-T_{0}}
$$

où : $C$ est une constante homogène à une température et $R$ la constante des gaz parfaits.

Ainsi la relaxation structurale observée lors $\mathrm{du}$ vieillissement et correspondant à une augmentation de la viscosité implique suivant la formule précédente un abaissement de $T_{\mathrm{gf}}$ (conformément au schéma de la figure 5).

Il est possible de vérifier que cette relation peut rendre compte d'une variation de la viscosité de deux ordres de grandeur : en effet, d'après les résultats présentés par Johari [11] la température $T_{0}$ peut être évaluée à $240 \mathrm{~K}$ environ; cette valeur entraîne une diminution de $T_{\mathrm{gf}}$ de $10 \mathrm{~K}$ lors du vieillissement. Par ailleurs, les courbes de la figure 4 suggèrent par leur déplacement vers les températures plus élevées que ce même vieillissement s'accompagne d'une augmentation de $T_{\mathrm{g}}$ (mesurée à température croissante) de 10 à $15 \mathrm{~K}$; ce point est tout à fait en accord avec le comportement classique des systèmes vitreux vers la zone de transition comme l'illustre la position des points $T_{\mathrm{g}}(\mathrm{B})$ et $T_{\mathrm{g}}(\mathrm{C})$ sur la figure 5 .

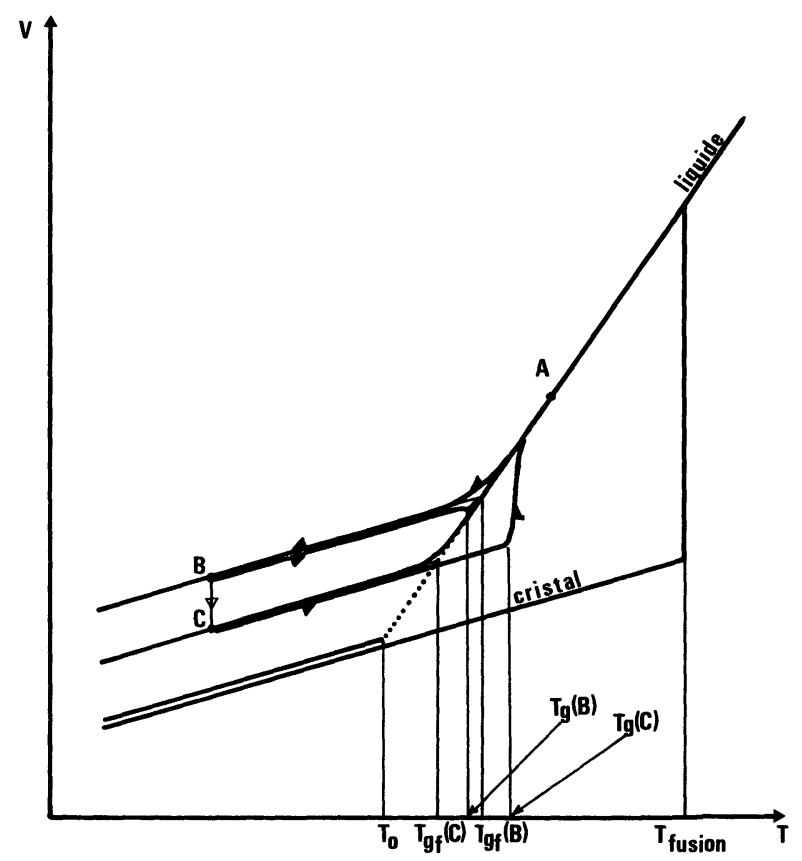

Fig. 5. - Représentation schématique de la variation du volume spécifique $v$ en fonction de la température.

[Schematic representation of the variation of the specific volume $v$ versus temperature.]

Examinons maintenant les résultats concernant le module dynamique (Fig. 3). La diminution du frottement intérieur au cours du vieillissement ne peut être attribuée à la seule variation de viscosité. En effet, l'étude du spectre de frottement intérieur $Q^{-1}=f(T)$ du sélénium vitreux [7] a montré l'existence de deux composantes :

- une augmentation monotone de frottement intérieur avec la température s'accélérant vers $T_{\mathrm{g}}$ ou composante $\alpha$, correspondant au mouvement irréversible d'une grande partie des éléments constituant le solide vitreux;

- une anomalie observée vers $-20,-30^{\circ} \mathrm{C}$ à $1 \mathrm{~Hz}$ ou composante $\beta$ correspondant au mouvement limité et réversible d'atomes ou de groupes d'atomes.

Sur la figure 1 l'équivalent de ces deux composantes apparaît clairement (les déformations correspondantes sont respectivement les déformations permanente $\varepsilon_{\mathrm{p}}$ et recouvrable $\varepsilon_{\mathrm{r}}$ ), et toutes deux sont affectées par le vieillissement. A partir de cette observation, on doit s'attendre à un effet de vieillissement sur les deux composantes du spectre $Q^{-1}=f(T)$ [7]; cette remarque est à rapprocher de celle de Goldstein [12] qui signale que dans le cas du sélénium vitreux les mouvements limités de groupes d'atomes pourraient contribuer à l'excès d'entropie dans une proportion non négligeable. Si la figure 2 souligne effectivement une évolution de la composante $\alpha$ la figure 4 montre toutefois au niveau de l'anomalie $\beta$ une variation relativement faible : cela peut être expliqué par le fait qu'il existe probablement une distribution des temps de relaxation du phénomène $\beta$ et que le vieillissement affecte surtout les éléments relaxants à longs temps 


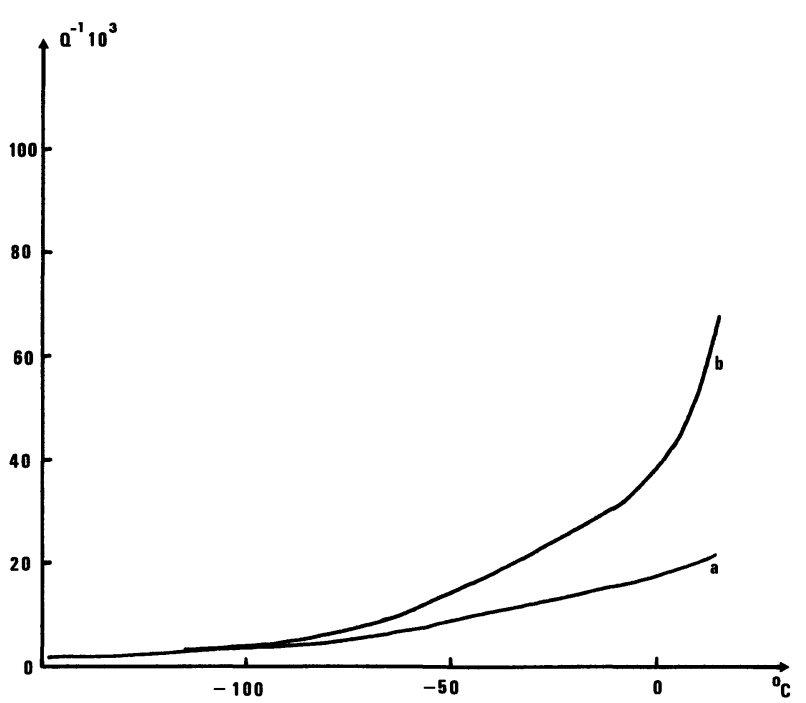

Fig. 6. - Spectre de frottement intérieur mesuré à la fréquence de $1 \mathrm{~Hz}$, l'échantillon de sélénium étant dans l'état vieilli (a) ou dans l'état trempé (b).

[Internal friction spectrum measured at $1 \mathrm{~Hz}$ with aged (a) and quenched (b) material.]

de relaxation ; ainsi au cours du vieillissement l'évolution de la partie recouvrable $\varepsilon_{\mathrm{r}}$ de la déformation est plus importante (Fig. 1) que celle du frottement intérieur dans le domaine $-20,-60{ }^{\circ} \mathrm{C}$ (Fig. 4) dans lequel le phénomène $\beta$ serait prépondérant [7]. Sur la figure 6, nous avons porté les courbes $Q^{-1}=f(T)$ mesurées plus classiquement au pendule de torsion à une fréquence de l'ordre de $1 \mathrm{~Hz}$. Les résultats sont qualitativement semblables à ceux de la figure 4, mais la variation liée au vieillissement est moins importante, justifiant par là même l'utilisation de notre technique basse fréquence. On peut noter, en outre, que l'anomalie (phénomène $\beta$ ) est plus ou moins nette suivant l'état de vieillissement du sélénium, et cela pourrait être à l'origine des différences observées entre les divers résultats [7, 13].

La discussion précédente nous conduit à décrire l'évolution des propriétés du sélénium vitreux lors du vieillissement en termes de diminution de volume libre (ou d'entropie configurationnelle). Un tel effet de vieillissement pourrait alors être un phénomène tout à fait général comme tendent à le montrer des résultats semblables obtenus dans le cas des verres d'oxydes [14] ou des verres organiques [15]. Toutefois, dans le cas du sélénium vitreux, nous devons rappeler l'hypothèse de la transformation des anneaux en chaînes lors du chauffage à $70^{\circ} \mathrm{C}$; le vieillissement incluerait alors un processus de dépolymérisation [6]. Cette idée, proche de celle relative à l'évolution de la réaction équilibrée chaînes-anneaux [4] implique toutefois, dans la mesure où les effets de relaxation structurale observés par méthode calorimétrique et par méthodes mécaniques ont la même origine, que la viscosité du sélénium augmente avec la fraction d'anneaux ; cela n'est pas en accord avec les remarques de divers auteurs $[16,17]$ : le contraire est même envisagé dans le cas du soufre [17]. En outre, dans l'hypothèse de l'évolution monotone de l'équilibre, il faudrait admettre d'après le modèle de Stephens [4] que les variations de propriétés mécaniques que nous avons observées (en particulier la variation de viscosité de deux ordres de grandeur) résultent d'une variation de la fraction d'anneaux qui évoluerait seulement entre $65 \%$ et $75 \%$ environ.

On doit noter que les phénomènes de vieillissement discutés ci-dessus entraînent un déplacement vers les temps longs de la courbe de distribution des temps de relaxation structurale. En fonction de cette remarque, nous envisageons une étude plus systématique de l'évolution des temps de relaxation mécanique associés à nos expériences.

En conclusion, nos mesures portant sur les propriétés micromécaniques du sélénium vitreux révèlent un phénomène d'évolution structurale vers $T_{\mathrm{g}}$ que l'on peut décrire, au moins formellement en utilisant les notions de température fictive et de volume libre. Une telle description peut rendre compte qualitativement des résultats expérimentaux sans qu'à priori soit nécessairement invoquée une quelconque modification de la nature ou de la forme des unités structurales. Cette évolution structurale du sélénium vitreux, d'ailleurs également observée par mesures ultrasonores $[18,19]$, pourrait être assimilée à celle observée dans d'autres systèmes vitreux (organiques ou minéraux).

\section{Bibliographie}

[1] Chang, R., Appl. Phys. Lett. 1 (1965) 231

[2] Winter, A., J. Can. Ceram. Soc. 41 (1972) 27.

[3] Das, G. C., Bever, M. B., Uhlman, D. R. and Moss, S. C., J. Non-Cryst. Solids 7 (1972) 251.

[4] Stephens, R. B., J. Non-Cryst. Solids 20 (1976) 75.

[5] Swiatkowski, W., Rocz. Chem. 47 (1973) 367.

[6] Cornet, J. et Rossier, D., Proceed. 5th Intern. Conf. Amorphous and liquid semiconductors, Ed. Stuke, J. and Breming, W., 1 (1973) 267.

[7] Etienne, S. et Perez, J., Revue Phys. Appl. 12 (1977) 837.

[8] ETIENNE, S., en cours de publication.

[9] HARRISON, G., The dynamic properties of supercooled liquids (Acad. Press, New York) 197623.

[10] Chen, H. S., J. Non-Cryst. Solids 22 (1976) 135.
[11] JohaRI, G. P., Proceed. Workshop on the glass transition and nature of the glassy state, Ed. Simha, R. and Goldstein, M. (New York Acad.) (in press).

[12] Goldstein, M., Non crystalline solids, Ed. Frischat, G. H., (Trans. Tech. Public.) 1977391.

[13] Struik, L. C. E., Physical ageing in amorphous polymers and other materials (Elsevier Sci. Publis. Co) 197822.

[14] Rötger, H., Amorphous materials, Ed. Douglas, R. W. and Ellis, B. (Willey Intersc.) 1972125.

[15] Kovacs, A. J., Adv. Polymer Sci. 3 (1964) 394.

[16] StePhens, R. B., Tech. Report. Office of Naval Research, N00014-77-C-0002 (1977).

[17] Eisenber G, A. and Tobolsky, A. V., J. Polym. Sci.46(1960) 19.

[18] Kittinger, E., J. Non-Cryst. Solids 27 (1978) 421.

[19] Etienne, S., Guenin, G. et Perez, J., Soumis à publication. 